\title{
COMPLETION THEOREM FOR COHOMOLOGICAL DIMENSIONS
}

\author{
WOJCIECH OLSZEWSKI
}

(Communicated by James E. West)

\begin{abstract}
We prove that for every separable metrizable space $X$ with $\operatorname{dim}_{G} X \leq n$, there exists a metrizable completion $Y$ of $X$ with $\operatorname{dim}_{G} Y \leq n$ provided that $G$ is either a countable group or a torsion group, and with $\operatorname{dim}_{G} Y \leq n+1$ if $G$ is an arbitrary group.
\end{abstract}

\section{INTRODUCTION}

This paper was inspired by the general plan to construct cohomological dimension theory parallel to the theory of covering dimension. We restrict our considerations to metrizable spaces.

We call a space $Y$ an absolute extensor of a space $X$ if every mapping $f: A \rightarrow Y$, where $A$ is a closed subset of $X$, extends over $X$; we write then $Y \in A E(X)$. We define the cohomological dimension $\operatorname{dim}_{G} X$ of a space $X$ with a coefficient group $G$ as

$$
\min \{n: K(G, n) \in A E(X)\},
$$

where $K(G, n)$ stands for the Eilenberg-Mac Lane CW complex (see, for example, [5]). Note that Y. Kodama proved in [3] that every mapping $f: X \rightarrow K$ from a closed subset $A$ of a metrizable space $X$ to a CW complex $K$ extends over an open set $U \subseteq X$ containing $A$. For a deeper discussion of cohomological dimensions in the realm of metrizable spaces we refer the reader to [1] and [2].

It is known as the completion theorem for covering dimension that for every metrizable space $X$ with $\operatorname{dim} X=n$, there exists a completely metrizable space $Y$ containing $X$ with $\operatorname{dim} Y=n$. In [4] L. R. Rubin and P. J. Schapiro proved that the analogous theorem holds for cohomological dimension with the coefficient group $G=\mathbb{Z}$, where $\mathbb{Z}$ denotes integers, provided $X$ is metrizable separable; they derived this theorem from their generalization of the EdwardsWalsh theorem.

We give the direct proof of that completion theorem and its generalization to every group $G$ which is either countable or torsion. We also prove that for any group $G$ and separable metrizable space $X$ with $\operatorname{dim}_{G} X=n$, there exists a completely metrizable space $Y$ containing $X$ with $\operatorname{dim}_{G} Y=n+1$. The

Received by the editors June 10, 1993 and, in revised form, August 13, 1993.

1991 Mathematics Subject Classification. Primary 54F45. 
results show that Conjecture 3 of [2] is true, and answer partially Problems 3 and 4.

\section{Proofs}

We shall start with the main theorem.

Theorem 1. Let $K$ be a countable $C W$ complex. For every metrizable separable space $X$ such that $K \in A E(X)$, there exists a metrizable completion $Y$ of $X$ such that $K \in A E(Y)$.

The following simple lemma established by Walsh will play a key role in the proof.

Lemma 1 (Walsh [5]). Every mapping $f: A \rightarrow K$ from a subspace $A$ of a metrizable separable space $Z$ to a $C W$ complex $K$ is homotopic to a mapping $f^{*}: A \rightarrow K$ which extends over an open subspace $U \subseteq Z$ containing $A$.

Lemma 2. Let $K$ be a $C W$ complex, $Z$ a metrizable separable space, and $X$ its subspace such that $K \in A E(X)$. Every mapping $g: C \rightarrow K$ from a closed subset $C \subseteq Z$ extends over an open set $U \subseteq Z$ such that $C \cup X \subseteq U$.

Proof. Extend $g$ to a $g^{*}$ over the closure of an open $V$ containing $C$, and next, $g^{*} \mid \mathrm{bd} V \cap X$ to an $h$ over $X$. It is easily seen that $f(x)=g^{*}(x)$ for $x \in V$ and $f(x)=h(x)$ for $x \in X-V$ is a well-defined mapping of $V \cup X$. Therefore by Lemma 1, there exists a mapping homotopic to $f$ which is extendable over an open set $U \subseteq Z$ such that $C \cup X \subseteq V \cup X \subseteq U$, and so is $g$.

Proof of Theorem 1. Assume that $X \subseteq Q$, where $Q$ stands for the Hilbert cube; we shall construct a $G_{\delta}$-subspace $Y \subseteq Q$ containing $X$ such that $K \in A E(Y)$. To this end, we shall first define inductively countable families $\mathfrak{U}_{n}$ of open subspaces of $Q$ containing $X$ and families of mappings $\left\{f_{U}: U \in \mathfrak{U}_{n}\right\}$, where $f_{U}: U \rightarrow K$.

Let $\left\{D_{k}: k \in \mathbb{N}\right\}$ be a family of closed subsets of $Q$ with the property that for any closed $C \subseteq Q$ and open $V \subseteq Q$ containing $C$, there is a $k \in \mathbb{N}$ such that $C \subseteq$ int $D_{k} \subseteq D_{k} \subseteq V$. The set $\left[D_{k}, K\right]$ of all homotopy classes of mappings from $D_{k}$ to $K$ is countable; that is, $\left[D_{k}, K\right]=\left\{\left[g_{k, i}^{(0)}\right]: i \in \mathbb{N}\right\}$. By Lemma 2, every $g_{k, i}^{(0)}$ extends over an open subspace $U_{k, i}^{(0)} \subseteq Q$ containing $D_{k} \cup X$; denote the extension by $f_{k, i}^{(0)}$. Set

$$
\mathfrak{U}_{0}=\left\{U_{k, i}^{(0)}: k, i \in \mathbb{N}\right\},
$$

and $f_{U}=f_{k, i}^{(0)}$ for $U=U_{k, i}^{(0)}$. By the Homotopy Extension Theorem, we have every mapping $f: D_{k} \rightarrow K$ extends over $U$ to a mapping homotopic to $f_{U}$ for some $U \in \mathfrak{U}_{0}$.

Suppose $\mathfrak{U}_{n}$ and mappings $f_{U}, U \in \mathfrak{U}_{n}$, are constructed. For every $U \in \mathfrak{U}_{n}$, $D_{l}, D_{m}, D_{p}$, and $D_{q}$ such that $D_{l} \subseteq$ int $D_{m}, D_{p} \subseteq$ int $D_{q}$, and $D_{q} \cap D_{l} \subseteq U$, consider the set

$$
\left[\left(D_{l} \cap U\right) \cup\left(U-\operatorname{int} D_{q}\right)\right] \cup\left(D_{p} \cap D_{m}\right) ;
$$

arrange these sets into a sequence $D_{k}^{(n+1)}, k \in \mathbb{N}$. Observe that for every $D_{k}^{(n+1)}=\left[\left(D_{l} \cap U\right) \cup\left(U-\operatorname{int} D_{q}\right)\right] \cup\left(D_{p} \cap D_{m}\right)$, we have $\left[\left(D_{l} \cap U\right) \cup\left(U-\operatorname{int} D_{q}\right)\right] \cap$ 
$\left(D_{p} \cap D_{m}\right)=D_{p} \cap D_{l}$, and $D_{k}^{(n+1)}$ is a closed subset of $Z_{k}=U \cup$ int $D_{q}$; recall that $D_{q} \cap D_{l} \subseteq U$. There is only a countable number of mappings $h_{k, i}: D_{p} \cap D_{m} \rightarrow$ $K, i \in \mathbb{N}$, such that $h_{k, i}\left|D_{p} \cap D_{l}=f_{U}\right| D_{p} \cap D_{l}$ up to homotopy relative $D_{p} \cap D_{l}$. Let $g_{k, i}^{(n+1)}(x)=h_{k, i}(x)$ for $x \in D_{p} \cap D_{m}$ and $g_{k, i}^{(n+1)}(x)=f_{U}(x)$ for $x \in\left[\left(D_{l} \cap U\right) \cup\left(U-\operatorname{int} D_{q}\right)\right]$. By Lemma $2, g_{k, i}^{(n+1)}$ extends over an open set $U_{k, i}^{(n+1)} \subseteq Z_{k}=U \cup \operatorname{int} D_{q}$ containing $X$; denote the extension by $f_{k, i}^{(n+1)}$.

Set $\mathfrak{U}_{n+1}=\left\{U_{k, i}^{(n+1)}: k, i \in \mathbb{N}\right\}$, and $f_{U}=f_{k, i}^{(n+1)}$ for $U=U_{k, i}^{(n+1)}$. By the Homotopy Extension Theorem, we have

every mapping $h: D_{k}^{(n+1)} \rightarrow K$ such that $h$ is homotopic to $f_{U}$ in $\left[\left(D_{l} \cap U\right) \cup\left(U-\operatorname{int} D_{q}\right)\right]$ extends over $V$ to a mapping homotopic to $f_{V}$ for some $V \in \mathfrak{U}_{n+1}$.

Let $Y=\bigcap_{n=0}^{\infty} \bigcap \mathfrak{U}_{n}$. We have to show that $K \in A E(Y)$, i.e., that every $f: A \rightarrow K$, where $A$ is a closed subset of $Y$, is extendable over $Y$. By virtue of Lemma 1 and the Homotopy Extension Theorem, we can assume that $f$ extends over an open set $U \subseteq Q$; denote the extension by the same letter. Consider a sequence of sets $D_{l(0)}, D_{l(1)}, \ldots$ such that $D_{l(n)} \subseteq$ int $D_{l(n+1)}$ for $n \in \mathbb{N}$ and $U=\bigcup\left\{D_{l(n)}: n \in \mathbb{N}\right\}$, and a sequence of sets $D_{p(0)}, D_{p(1)}, \ldots$ such that $D_{p(n+1)} \subseteq$ int $D_{p(n)}$ for $n \in \mathbb{N}$ and $\operatorname{cl}_{Q} A=\bigcap\left\{D_{p(n)}: n \in \mathbb{N}\right\}$.

We shall define inductively sets $U_{n} \in \mathfrak{U}_{n}$ and mappings $f_{n}: U_{n} \rightarrow K$ such that

$$
\begin{gathered}
D_{p(n)} \cap D_{l(n)} \subseteq U_{n}, \\
f_{n} \text { is homotopic to } f_{U_{n}}, \\
f_{n}(x)=f_{n+1}(x) \text { for every } x \in Y \cap\left[\left(Q-\operatorname{int} D_{p(n)}\right) \cup D_{l(n)}\right], \\
f_{n}(x)=f(x) \text { for } x \in D_{p(n)} \cap D_{l(n)} .
\end{gathered}
$$

Let $U_{0} \in \mathfrak{U}_{0}$ be such that $f \mid D_{l(0)}$ extends over $U_{0}$ to a mapping $f_{0}$ homotopic to $f_{U_{0}}$ (see (1)). Suppose $U_{n}$ and $f_{n}$ are defined. Let $D_{l}=D_{l(n)}$, $D_{m}=D_{l(n+1)}, D_{p}=D_{p(n+1)}, D_{q}=D_{p(n)}$. Consider $D_{k}^{(n+1)}=\left[\left(D_{l} \cap U_{n}\right) \cup\right.$ $\left.\left(U_{n}-\operatorname{int} D_{q}\right)\right] \cup D_{p} \cap D_{m}\left(D_{q} \cap D_{l} \subseteq U_{n}\right.$ by condition (3) of the inductive assumption), and the mapping $h: D_{k}^{(n+1)} \rightarrow K$ defined by letting $h(x)=f_{n}(x)$ for $x \in\left[\left(D_{l} \cap U_{n}\right) \cup\left(U_{n}-\operatorname{int} D_{q}\right)\right]$ and $h(x)=f(x)$ for $x \in D_{p} \cup D_{m}$. By virtue of (3) and (6) of the inductive assumption $h$ is well defined; by (2), $h$ extends over a $U_{n+1} \in \mathfrak{U}_{n+1}$ to a mapping $f_{n+1}$ homotopic to $f_{U_{n+1}}$. It is easily seen that (3)-(6) are satisfied.

By (5), the sequence $\left(f_{n}\right)_{n=0}^{\infty}$ determines a mapping $f^{*}: Y \rightarrow K$ which is an extension of $f$ by (6).

Corollary 1. Let $G$ be a countable abelian group. Every separable metrizable space $X$ has a completion $Y$ with $\operatorname{dim}_{G} Y=\operatorname{dim}_{G} X$.

In [1] J. Dydak established the following theorem.

Theorem. Let $G$ be an abelian group. Then

$$
\operatorname{dim}_{G} X=\max \left\{\operatorname{dim}_{\text {Tor } G} X, \operatorname{dim}_{G / \text { Tor } G} X\right\},
$$




$$
\operatorname{dim}_{G} X=\max \left\{\operatorname{dim}_{H} X: H \in \sigma(G)\right\},
$$

where $\sigma(G)$ is a countable family of countable groups, provided $G$ is a torsion group

there exists a countable group $H(G)$ such that $\operatorname{dim}_{G} X \leq$ $\operatorname{dim}_{H(G)} X \leq \operatorname{dim}_{G} X+1$ provided $G$ is torsion free

for every metrizable space $X$.

By virtue of this theorem, Corollary 1 and the monotonicity of cohomological dimension (see [5]), we obtain the following corollary.

Corollary 2. Let $X$ be a separable metrizable space, and $G$ an abelian group. Then there exists a completion $Y$ of $X$ with $\operatorname{dim}_{G} Y \leq \operatorname{dim}_{G} X+1$, and with $\operatorname{dim}_{G} Y=\operatorname{dim}_{G} X$ provided $G$ is a torsion group.

We do not know whether for any abelian group $G$ and separable metrizable space $X$ there exists a countable abelian group $H$ such that $\operatorname{dim}_{H} X \leq \operatorname{dim}_{G} X$ and $\operatorname{dim}_{G} Y \leq \operatorname{dim}_{H} Y$ for every separable metrizable space $Y$. Of course, the positive answer to this question would give the completion theorem for an arbitrary abelian group $G$.

\section{AdDED IN PROOF}

The author is indebted to L. R. Rubin for pointing out a mistake in the introduction; namely, his paper with P. J. Schapiro ([4]) contains the completion theorem for integer cohomological dimension under the assumption that $X$ is a metrizable, not necessarily separable, space.

\section{REFERENCES}

1. J. Dydak, Cohomological dimension and metrizable spaces, Trans. Amer. Math. Soc. 337 (1993), 219-234.

2. _ Cohomological dimension and metrizable spaces II, preprint.

3. Y. Kodama, Note on an absolute neighbourhood extensor for metric spaces, J. Math. Soc. Japan 8 (1956), 206-215.

4. L. R. Rubin and P. J. Schapiro, Cell-like maps onto non-compact spaces of finite cohomological dimension, Topology Appl. 27 (1987), 221-244.

5. J. J. Walsh, Dimension, cohomological dimension, and cell-like mappings, Lecture Notes in Math., vol. 870, Springer-Verlag, Berlin and New York, 1981, pp. 105-118.

Department of Mathematics, University of Warsaw, ul. Banacha 2, 02-097 Warszawa, POLAND

Current address: Department of Economics, University of Warsaw, ul. Dluga 44/50, 00-241 Warszawa, Poland

E-mail address: wojolsz@mimuw.edu.p1 